Nervenarzt 2022 · 93:599-604

https://doi.org/10.1007/s00115-021-01206-w

Angenommen: 13. September 2021

Online publiziert: 15 . Oktober 2021

(c) Der/die Autor(en) 2021

\section{Intrazerebrale Blutungen unter Plättchenaggregationshemmung und oraler Antikoagulation bei Patienten mit zerebraler Amyloidangiopathie}

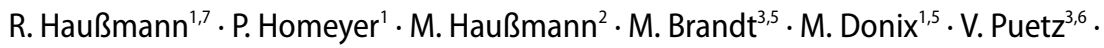 \\ J. Linn ${ }^{4,6}$ \\ ${ }^{1}$ Klinik und Poliklinik für Psychiatrie und Psychotherapie, Universitätsklinikum Carl Gustav Carus, \\ Technische Universität Dresden, Dresden, Deutschland \\ ${ }^{2}$ Dialysepraxis Leipzig, MVZ, Leipzig, Deutschland \\ ${ }^{3}$ Klinik und Poliklinik für Neurologie, Universitätsklinikum Carl Gustav Carus, Technische Universität \\ Dresden, Dresden, Deutschland \\ ${ }^{4}$ Institut und Poliklinik für diagnostische und interventionelle Neuroradiologie, Universitätsklinikum Carl \\ Gustav Carus, Technische Universität Dresden, Dresden, Deutschland \\ ${ }^{5}$ DZNE, Deutsches Zentrum für Neurodegenerative Erkrankungen, Dresden, Deutschland \\ ${ }^{6}$ DNVC, Dresdner Neurovaskuläres Centrum, Dresden, Deutschland \\ ${ }^{7}$ Universitäts DemenzCentrum (UDC), Klinik und Poliklinik für Psychiatrie und Psychotherapie, \\ Uniklinikum Dresden, Dresden, Deutschland
}

\title{
Zusammenfassung
}

Die Durchführung einer oralen Antikoagulation ist bei Patienten mit einer zerebralen Amyloidangiopathie eine therapeutische Herausforderung. Die Assoziation der zerebralen Amyloidangiopathie mit Lobärblutungen, eine hohe Mortalität intrazerebraler Blutungen insbesondere unter oraler Antikoagulation sowie das hohe Rezidivrisiko solcher Blutungen erfordern eine strenge und interdisziplinäre Risiko-Nutzen-Abwägung. Vitamin-K-Antagonisten erhöhen das Risiko für die mit intrazerebralen Blutungen vergesellschaftete Mortalität um $60 \%$ und sollten daher möglichst vermieden bzw. speziellen klinischen Situationen (z.B. mechanischer Aortenklappenersatz) vorbehalten sein. Auch der Einsatz von neuen oralen Antikoagulanzien und Thrombozytenaggregationshemmern bedarf einer strengen Risiko-Nutzen-Abwägung, da auch diese Substanzen das zerebrale Blutungsrisiko erhöhen. Insbesondere bei Patienten mit einer absoluten Arrhyhtmie bei Vorhofflimmern ist der interventionelle Vorhofohrverschluss eine therapeutische Alternative. Darüber hinaus sind weitere klinische Implikationen bei Patienten mit zerebraler Amyloidangiopathie Gegenstand dieser Literaturübersicht, beispielsweise Besonderheiten nach akutem ischämischem Schlaganfall und erforderlicher Sekundärprophylaxe, bei vorherigen intrazerebralen Blutungen und bei Patienten mit kognitiven Defiziten.

\section{Schlüsselwörter}

Cerebrale Amyloidangiopathie · Intracerebrale Blutung · Thrombozytenaggregationshemmung · Orale Antikoagulation · Interventioneller Vorhofohrverschluss 


\section{Hintergrund}

Eine zerebrale Amyloidangiopathie (CAA) ist bei $20-40 \%$ der älteren Bevölkerung ohne Demenzerkrankung und bei 50-60\% der Menschen mit Demenzerkrankung nachweisbar [1]. Die CAA kann sich mit intrazerebralen Lobärhämatomen, zerebralen Mikroblutungen, fokalen Subarachnoidalblutungen und einer kortikalen superfiziellen Siderose (CSS) manifestieren. Bei der CSS handelt es sich um lineare Hämosiderinablagerungen im Subarachnoidalraum oder den oberflächlichen Kortexschichten der Konvexität, die Residuen vorangegangener fokaler Subarachnoidalblutungen darstellen. Die CAA wird bei einem beträchtlichen Anteil der Fälle nichttraumatischer intrazerebraler Blutungen (ICB) als ursächlich angenommen [1]. Patienten mit einer CAA und stattgehabter ICB haben ferner ein erhöhtes Rezidivrisiko für eine erneute ICB [2]. Die bedeutsamsten Prädiktoren für die Entwicklung einer ICB bei Patienten mit einer CAA sind eine zurückliegende ICB, das Vorliegen einer disseminierten CSS sowie die Dauer und Art der oralen Antikoagulation [2]. Insbesondere bei bestehender Indikation zur therapeutischen Antikoagulation älterer Patienten, beispielsweise aufgrund eines Vorhofflimmerns, sehen sich Behandler häufig mit dem Dilemma konfrontiert, dass die aktuell verfügbaren Assessments zur Abschätzung des Blutungsrisikos unter Antikoagulation, wie beispielsweise der HAS-BLED-Score [3], das Vorliegen einer CAA und das damit assoziierte ICBRisiko unzureichend berücksichtigen und momentan eine geringe Evidenz zur Risiko-Nutzen-Abwägung hinsichtlich der Einleitung oder des Wiederbeginns einer oralen Antikoagulation bei diesen Patienten besteht.

Etwa 9\% der über 65-jährigen Menschen leiden an einer absoluten Arrhythmie bei Vorhofflimmern, und sowohl die Prävalenz der CAA als auch die Prävalenz des Vorhofflimmerns steigen im höheren Lebensalter deutlich an $[4,5]$, weshalb dieses Dilemma von wachsender klinischer Bedeutung ist. Vor diesem Hintergrund fasst diese Literaturübersichtsarbeit aktuelle Daten zum Blutungsrisiko bei Patien- ten mit CAA und gerinnungshemmender Medikation zusammen.

\section{Methodik}

Diese Übersichtsarbeit wurde unter Nutzung der über PubMed verbundenen biomedizinischen Datenbanken erstellt. Eine zusätzliche Suche erfolgte anhand der in den Literaturverzeichnissen gefundenen Original- und Übersichtsarbeiten. Dabei wurden präferenziell Originalarbeiten gesucht. Auch die verfügbaren Leitlinien wurden berücksichtigt. In Anbetracht der Datenlage fanden jedoch verschiedene Studiendesigns, metaanalytische Daten und auch Literaturübersichten Berücksichtigung.

Risiko für intrazerebrale Blutungen bei zerebraler Amyloidangiopathie allgemein

Die CAA wird zunehmend als wichtige Ursache für eine lobäre ICB im höheren Lebensalter wahrgenommen [6]. Das primäre ICB-Risiko bei Patienten mit einer CAA ist abhängig von verschiedenen MRT-Biomarkern und von der allgemeinen Amyloidlast [4]. Lobäre Hämorrhagien auf dem Boden einer CAA weisen eine Mortalität von $20-30 \%$ auf $[7,8]$. Insbesondere ICBs unter oraler Antikoagulation sind mit einer hohen Mortalität von bis zu $52 \%$ vergesellschaftet [4]. Eine Hämatomgröße $<50 \mathrm{ml}$ und eine GCS-Score $\geq 8$ sind in diesem Zusammenhang bedeutsame Prädiktoren für eine günstige Prognose [7].

Patienten mit einer CAA und vorheriger ICB weisen ein jährliches Rezidivblutungsrisiko von 8,9\% pro Jahr auf [4]. Insbesondere im Vergleich zu Patienten mit einer hypertensiv bedingten ICB weisen Patienten mit einer CAA-assoziierten ICB ein vergleichsweise hohes Rezidivrisiko auf [9]. In zwei Fallserien lag die Rezidivblutungsrate nach 2 Jahren bei $21 \%$ bzw. nach 2,6 Jahren bei $24 \%[10,11]$. Andere Studien zeigen ein ungefähres Rezidivblutungsrisiko bei hypertensiver ICB von 7\% [12]. Aktuelle Daten lassen vermuten, dass das Rezidivblutungsrisiko wiederum mit jeder weiteren ICB ansteigt [13]. Insbesondere Areale mit hoher Amyloidlast scheinen dabei Prädilektionsstellen für weitere ICBs zu sein $[8,14]$.
Als Risikofaktoren für eine ICB im Rahmen einer CAA gelten eine stattgehabte ICB (HR 7,7; 95\%-KI 1,4-15,7), das Vorliegen einer CSS, eine höhere Anzahl lobärer Mikroblutungen und CT-Hypodensitäten im posterioren Marklager [13]. Das mit kortikalen Mikroblutungen assoziierte ICB-Risiko nimmt mitzunehmender Anzahl der Mikroblutungen zu [4]. Bezüglich der cSS konnte eine Metaanalyse von 6 Studien mit > 1200 symptomatischen Patienten zeigen, dass das jährliche ICB-Risiko bei Patienten in Abhängigkeit des Vorliegens und Schweregrades einer CSS ansteigt: ohne cSS 3,9\% pro Jahr (95\%-KI 1,7-6,1\%), mit cSS 11,1 \% pro Jahr (95\%-KI 7-15,2\%; [15]). Bei Patienten mit einer fokalen cSS ( $<4$ betroffene kortikale Furchen) betrug das ICB-Risiko 9,1\% pro Jahr, während es bei den Patienten mit einer disseminierter CSS ( $\geq 4$ Furchen) $12,5 \%$ pro Jahr betrug [15]. Somit ist neben dem Vorliegen einer CSS auch der Ausprägungsgrad (fokal vs. disseminiert) als zusätzlicher Risikofaktor für eine ICB zu betrachten (HR fokale CSS: 2,11 [KI] vs. HR disseminierte CSS: 4,28 [KI]; [15]). Andere Daten zeigen ein jährliches Blutungsrisiko von 19\% pro Jahr bei Patienten mit CAA und zurückliegender ICB, wenn bildmorphologisch fokale Subarachnoidalblutungen (fSAH), d. h. umschriebene Subarachnoidalblutungen im Bereich der Konvexitäten der Großhirnhemisphären, gefunden werden [4].

\section{Risiko für intrazerebrale Blutungen bei CAA unter Thrombozyten- aggregationshemmung}

Die Therapie mit Thrombozytenaggregationshemmern war in einer populationsbasierten Querschnittstudie mit 1062 Patienten $>60$ Jahre mit einer höheren Prävalenz zerebraler Mikroblutungen assoziiert [16]. Patienten unter antithrombotischer Medikation wiesen mehr zerebrale Mikroblutungen auf als Patienten ohne thrombozytenaggregationshemmende Therapie (adjustierte OR 1,71; $95 \%$-KI 1,21-2,41), wobei dieser Zusammenhang für orale Antikoagulanzien in der untersuchten Kohorte nicht gefunden wurde (adjustierte OR 1,49; $95 \%-K I ~ 0,82-2,71$; [16]). Insbesondere die Häufigkeit strikt lobär lokalisierter zerebraler Mikroblutungen war unter Behandlung mit ASS erhöht, was eine differenzi- 
Tab. 1 Gegenüberstellung von Risikofaktoren für ICB bei CAA und thrombembolische Komplikationen

\begin{tabular}{|c|c|}
\hline Risikofaktoren für ICB bei CAA & $\begin{array}{l}\text { Risikofaktoren für thrombembolische Kompli- } \\
\text { kationen }\end{array}$ \\
\hline Z.n. ICB & $\begin{array}{l}\text { Tumorassoziierte und andere Hyperkoagulopa- } \\
\text { thien }\end{array}$ \\
\hline Disseminierte kortikale superfizielle Siderose & Z.n. mechanischem Klappenersatz \\
\hline \multirow{2}{*}{$\begin{array}{l}\text { Antikoagulation mit Phenprocoumon, } \\
\text { (N)OAK, NSAR - insbesondere bei länge- } \\
\text { rer Therapiedauer }\end{array}$} & (Z.n.) tiefe(r) Beinvenenthrombose \\
\hline & Vorhof- oder Ventrikelthrombus \\
\hline Hohe Anzahl lobärer Mikroblutungen & Vorhofflimmern \\
\hline Hohe Amyloidlast & Z.n. zerebraler Ischämie \\
\hline
\end{tabular}

elle Beeinflussung des Blutungsrisikos bei Patienten mit einer CAA suggeriert [16]. Um das „Confounding-by-indication“-Bias möglichst gering zu halten, erfolgten in dieser Analyse eine Adjustierung für das kardiovaskuläre Risiko und ein Ausschluss von Patienten mit bekannter zerebrovaskulärer Erkrankung.

Nach stattgehabter ICB und nach Kontrolle für sonstige klinische ICB-Prädiktoren erhöht ASS in multivariaten Analysen das Risiko für eine Rezidivblutung (HR 3,95, $95 \%-K I 1,6-8,3 ; p=0,021 ;[13])$. Bei Patienten nach ICB war der Wiederbeginn einer Thrombozytenaggregationshemmung bei Patienten mit einer CAA jedoch nicht mit einem erhöhten Rezidiv-ICB-Risiko vergesellschaftet [17]. Relevante Limitationen der bisherigen Daten zu Assoziationen zwischen zerebralen Mikroblutungen und bestehender Thrombozytenaggregationshemmung sind das oft retrospektive Studiendesign, welches die Beschreibung von Kausalzusammenhängen begrenzt, die Tatsache, dass Hämosiderinablagerungen für eine nicht näher definierte Zeitdauer nachweisbar bleiben und somit möglicherweise vorbestehende Mikroblutungen in den durchgeführten Untersuchungen Ergebnisse zu entsprechenden Assoziationen verfälschen sowie eine Verzerrung dadurch, dass insbesondere Patienten mit erhöhtem kardiovaskulärem Risiko, die a priori auch ein erhöhtes Risiko für zerebrale Mikroblutungen aufweisen, eine thrombozytenenaggregationshemmende Therapie erhalten [16]. Eine kanadische Machbarkeitsstudie in 2 Phasen (NASPAF$\mathrm{ICH} ; 1$. Phase: Ausschluss von Patienten mit CAA, 2. Phase Einschluss von Patienten mit (AA) mit den kombinierten Endpunk- ten ischämischer Schlaganfall und ICB nach Wiederbeginn von ASS oder NOAK zeigte bei bislang 30 eingeschlossenen Patienten (9 mit ASS, 21 mit NOAK) unter striktem Blutdruckprotokoll $(\mathrm{RR}<130 / 80)$ keine erneuten ICBs bei einem ischämischen Schlaganfall in der ASS-Gruppe [18, 19].

\section{Risiko für intrazerebrale Blutungen unter oraler Antikoagulation}

In einer Untersuchung von 6045 Patienten > 55 Jahre mit Vorhofflimmern und durchschnittlich 6-jähriger Behandlungsdauer mit oraler Antikoagulation oder Thrombozytenaggregationshemmung entwickelten 74 Patienten (1,22\%) eine nichttraumatische ICB, von denen $51,4 \%$ die diagnostischen Kriterien einer CAA erfüllten [20]. Die Mortalität zwischen Patienten mit und ohne Zeichen einer CAA unterschied sich nicht (orale Antikoagulanzien: 10-Jahres-Mortalität CAA-Gruppe $45 \%$ vs. $63 \%$ in Nicht-CAAGruppe $[p=0,46]$; Thrombozytenaggregationshemmung: 10-Jahres-Mortalität CAA-Gruppe $79 \%$ vs. $70 \%$ Nicht-CAAGruppe $[p=0,66])$. Vor allem unter Therapie mit Vitamin-K-Antagonisten ist das Auftreten einer ICB mit dem Vorhandensein zerebraler Mikroblutungen assoziiert, was eine Risikoerhöhung für Vitamin K-Antagonisten-assoziierte ICBs suggeriert [21]. Das höchste Risiko besteht unter Therapie mit Phenprocoumon bzw. Warfarin, gefolgt von neuen oralen Antikoagulanzien (NOAK) und Thrombozytenaggregationshemmern [2]. Insbesondere die orale Antikoagulation mit Vitamin-KAntagonisten erhöht das ICB-Risiko bei
Patienten mit einer CAA um den Faktor 7 bis 10 und die ICB-assoziierte Mortalität um $60 \%$ [22-24]. Eine prospektive Beobachtungsstudie bei Patienten mit neu diagnostiziertem Vorhofflimmern, neu begonnener oraler Antikoagulation, Baseline-Schädel-MRT und einem Follow-up nach 24 Monaten mit 1490 Patienten (CROMIS-2-Studie) zeigte insgesamt 14 ICBs im Beobachtungszeitraum (11 hemisphärielle Blutungen, 1 Subduralhämatom, 1 Subarachnoidalblutung; [25]). Im initialen MRT wiesen $21 \%$ kortikale Mikroblutungen und $<1 \%(n=5)$ eine cSS auf; 3\% erfüllten die modifizierten Boston-Kriterien für die Diagnose einer wahrscheinlichen CAA. Die Patienten der ICB-Gruppe litten häufiger an einem Diabetes mellitus, waren häufiger auf einen Vitamin-K-Antagonisten als auf NOAK eingestellt (12 vs. 2) und wiesen mehr kortikale Mikroblutungen und cSS auf. Insbesondere das Vorliegen von Mikroblutungen erhöhte das Blutungsrisiko im Vergleich zu Patienten ohne kortikale Mikroblutungen um den Faktor 3. Diese Untersuchung liefert einen wichtigen Beitrag zur besseren Prädiktion einer ICB im Rahmen eines kombinierten Modells unter Berücksichtigung von HAS-BLEDScores, kortikalen Mikroblutungen, Diabetes mellitus und Art der Antikoagulation.

\section{Klinische Implikationen}

Vor dem Hintergrund des erhöhten Risikos für spontane Lobärblutungen bei Patienten mit einer CAA unter oraler Antikoagulation bedarf es einer individualisierten Risiko-Nutzen-Abwägung vor der Behandlung mit Thrombozytenaggregationshemmern und oralen Antikoagulanzien. Entscheidend im Rahmen dieser Abwägung sind stets die Stärke der jeweiligen Indikation, das individuelle ICB-Risiko sowie die Verfügbarkeit alternativer Therapieoptionen [2]. In derartigen Situationen sollten daher bestehende Risikofaktoren für intrazerebrale Blutungen und thrombembolische Ereignisse gegenübergestellt und eine individuelle Nutzen-Risiko-Abwägung getroffen werden (ס Tab. 1).

Insbesondere beim komorbiden Vorliegen einer CAA und einer absoluten Arrhythmie bei Vorhofflimmern ist das therapeutische Management Gegenstand kon- 
troverser Diskussionen und bislang mit unzureichender Evidenz unterfüttert [26]. Der interventionelle Verschluss des linken Vorhofohres stellt eine mögliche therapeutische Alternative dar. Kleinere Beobachtungsstudien und eine Metaanalyse von 19 RCTs aus dem Jahre 2017 deuten darauf hin, dass es sich beim Vorhofohrverschluss um eine therapeutische Maßnahme mit einer ähnlichen Effektivität wie die der oralen Antikoagulation handelt [26, 27]. Die 5-Jahres-Nachbeoachtung der PROTECTAF- und PREVAIL-Studie, die die Wirksamkeit von Vitamin-K-Antagonisten und interventionellem Vorhofohrverschluss verglichen, zeigte signifikant weniger ICBs bei einer vergleichbaren Prävalenz ischämischer Schlaganfälle [28].

\section{Wiederbeginn einer oralen Antikoagulation nach ICB}

Bei Patienten nach stattgehabter ICB unter Antikoagulanzientherapie stellt sich im klinischen Kontext häufig die Frage, ob eine vorbestehende orale Antikoagulation wieder aufgenommen werden sollte. Neben einem unzureichend behandelten Hypertonus, einem hohen Lebensalter, der Notwendigkeit einer dualen Thrombozytenaggregationshemmung und einer erlittenen ICB sprechen auch multiple zerebrale Mikroblutungen gegen einen Wiederbeginn der oralen Antikoagulation [1]. Sowohl für Vitamin-K-Antagonisten als auch für NOAKs stellt eine zurückliegende ICB formal eine Kontraindikation für eine weitere Gabe dar. Dennoch profitieren Patienten auch in dieser klinischen Konstellation wahrscheinlich vom Wiederbeginn der oralen Antikoagulation und auch vom Wiederbeginn einer vorbestehenden Thrombozytenaggregationshemmung [17, 29].

Eine Metaanalyse mit Daten aus drei Registerstudien mit insgesamt 633 Patienten mit nichtlobärer und 379 Patienten mit lobärer antikoagulanzienassoziierter ICB zeigte, dass die Wiederaufnahme der oralen Antikoagulation mit einer signifikant geringeren Mortalität, einem besseren klinisch-funktionellen Outcome und einer signifikant reduzierten Rate ischämischer Schlaganfälle bei nicht erhöhtem ICB-Risiko, sowohl hinsichtlich kortikaler als auch hinsichtlich subkortikaler ICBs, assoziiert ist [30].
Der Nachweis einer CAA kann alternativ dazu führen, dass von einer erneuten oralen Antikoagulation abgesehen und ein Vorhofohrverschluss präferiert wird [1]. In welchem Ausmaß Patienten mit Vorhofflimmern nach einer stattgehabten ICB von einem interventionellen Vorhofohrverschluss profitieren, ist zum aktuellen Zeitpunkt jedoch nicht geklärt und Gegenstand laufender prospektiver Studien (z. B. STROKECLOSE-Studie; [31]).

Darüber hinaus sind begleitende kognitive Defizite bei bestehender Indikation zur oralen Antikoagulation zu berücksichtigen. Etwa $25 \%$ älterer Patienten mit Vorhofflimmern weisen bereits vor der ersten ICB kognitive Defizite auf [32], welche wiederum auch Assoziationen zur CAA aufweisen. Insbesondere bei Patienten mit Alzheimer-Demenz weisen Mikroblutungen auf eine Assoziationen zum Vorliegen einer CAA hin [33]. Aus diesem Grund sollten Patienten mit kognitivem Abbau, vorheriger ICB oder transienten Episoden fokal-neurologischer Defizite vor Beginn der Antikoagulation eine MRT des Neurokraniums erhalten, um das Vorhandensein kortikaler Mikroblutungen, einer cSS oder einer sSAH als mögliche Korrelate einer CAA zu erkennen [4]. Im Rahmen eines multidisziplinären Assessments und unter Berücksichtigung der Erkenntnisse aus der CROMIS-2-Studie sollte eine ausführliche Risiko-Nutzen-Abwägung hinsichtlich der Eindosierung einer oralen Antikoagulation erfolgen. Beim Vorliegen einer CAA sollten Vitamin-K-Antagonisten zugunsten des Einsatzes von NOAKs vermieden werden, sofern keine spezielle Indikation für den Einsatz von Vitamin-K-Antagonisten besteht (z.B. bei valvulärem Vorhofflimmern, Z.n. mechanischem Herzklappenersatz). Wenn der Einsatz eines NOAK klar indiziert ist, bedarf es vor allem bei älteren Patienten einer Reevaluation des Blutungsrisikos, insbesondere in Form eines engmaschigen Blutdruckmonitorings mit einem Blutdruckziel unter 130/80 mm Hg und gegebenenfalls einer zerebralen MRTKontrolle im Verlauf [4].

\section{Weitere therapeutische Aspekte}

Das Vorliegen einer CAA muss außerdem bei der akuten Schlaganfallbehandlung und der Sekundärprävention gewürdigt werden [34]. Das Vorhandensein und eine hohe Last an zerebralen Mikroblutungen sind unabhängige Prädiktoren für eine symptomatische ICB unter i.v. Thrombolysetherapie [35]. Insbesondere eine hohe Last an zerebralen Mikroblutungen ist im Kontext der i.v. Thrombolysetherapie mit einer erhöhten Mortalität und insbesondere bei älteren Patienten mit dem Auftreten lyseassoziierter ICBs verbunden [36], wobei das Vorhandensein von Mikroblutungen nach jetziger Studienlage trotz des erhöhten ICB-Risikos keine Kontraindikation für eine systemische Thrombolyse darstellt. In Grenzsituationen (z.B. geringes klinisches Defizit) kann das bekannte Vorliegen einer CAA jedoch nach unserer Ansicht in die individualisierte Entscheidungsfindung und NutzenRisiko-Abwägung zur Durchführung einer systemischen Lysetherapie einfließen.

Unter i.v. Thrombolysetherapie neu aufgetretene zerebrale Mikroblutungen sind annehmbar mit dem Vorliegen einer CAA vergesellschaftet [37]. Aktuell existieren jedoch keine gesonderten leitlinienbasierten Empfehlungen zur Behandlung des ischämischen Schlaganfalls bei Patienten mit schwerer Mikroangiopathie, Amyloidangiopathie oder Vorbehandlung mit Thrombozytenaggregationshemmern.

Im Bereich der Sekundärprävention ischämischer Schlaganfälle zeigen aktuelle Daten, dass eine konsequente antihypertensive Therapie mit einem Zielblutdruck < 130/80 mm Hg das Risiko für eine Rezidiv-ICB bei Patienten mit einer CAA deutlich reduzieren kann [38]. Ferner gilt es, aktuelle Daten hinsichtlich einer sekundärprophylaktischen Therapie mit Statinen zu berücksichtigen. Während ein allgemeiner Konsens hinsichtlich einer Inzidenzreduktion kardiovaskulärer Ereignisse unter Statintherapie besteht, wurde eine erhöhte Inzidenz von ICBs und insbesondere kortikosubkortikaler Mikroblutungen beobachtet, was auf eine mögliche Assoziationen zur CAA hindeutet $[34,39]$. Es wird kontrovers diskutiert, ob das Risiko einer Rezidivblutung nach ICB unter einer sekundärprophylaktischen Statintherapie ansteigt [40]. Neuere Daten zeigen, dass Statine bei Patienten nach Schlaganfall das ICB-Risiko zwar erhöhen, die präventiven Effekte hinsichtlich ischämischer Schlaganfälle insgesamt 
jedoch zu überwiegen scheinen [41]. Möglicherweise führt die frühe Initiierung einer Statintherapie bei Patienten mit lobärer ICB zu einer Vergrößerung des perihämorrhagischen Ödems [42]. Es bedarf jedoch weiterer Studien, um Statineffekte auf das ICB-Risiko differenziert beschreiben und antizipieren zu können.

\section{Fazit für die Praxis}

Das erhöhte ICB-Rezidivrisiko bei Patienten mit einer CAA unter Behandlung mit Thrombozytenaggregationshemmern und oralen Antikoagulanzien muss im klinischen Kontext Berücksichtigung finden. Klinische Szenarien, in denen eine bestehende Indikation zur oralen Antikoagulation mit dem Blutungsrisiko bei komorbid bestehender CAA abgewogen werden muss, bedürfen eines multidisziplinären Assessments und einer interdisziplinären Risiko-Nutzen-Abwägung unter Würdigung der individuellen Risikofaktoren für ICBs und thrombembolische Ereignisse. Da die Datenlage aktuell begrenzt ist, bleibt die Entscheidung für oder gegen eine orale Antikoagulation bei Patienten mit CAA zum gegenwärtigen Zeitpunkt eine Einzelfallentscheidung. Der interventionelle Vorhofohrverschluss kann in derartigen Situationen eine therapeutische Alternative darstellen. Außerdem sollte das Blutungsrisiko im Verlauf der Behandlung älterer Patienten kontinuierlich reevaluiert werden. Bei Patienten nach ICB, mit kognitivem Abbau oder zurückliegenden transienten Episoden neurologischer Defizite sollte vor Beginn der oralen Antikoagulation eine MRT des Neurokraniums erfolgen.

Korrespondenzadresse

PD Dr. med. habil. R. Haußmann

Universitäts DemenzCentrum (UDC), Klinik und Poliklinik für Psychiatrie und Psychotherapie, Uniklinikum Dresden

Dresden, Deutschland

robert.haussmann@ukdd.de

Funding. Open Access funding enabled and organized by Projekt DEAL.

\section{Einhaltung ethischer Richtlinien}

Interessenkonflikt. R. Haußmann, P. Homeyer, M. Haußmann, M. Brandt, M. Donix, V. Puetz und J. Linn geben an, dass kein Interessenkonflikt besteht.

Für diesen Beitrag wurden von den Autoren keine Studien an Menschen oder Tieren durchgeführt. Für die aufgeführten Studien gelten die jeweils dort angegebenen ethischen Richtlinien.

\section{Intracerebral hemorrhage under platelet inhibition and oral} anticoagulation in patients with cerebral amyloid angiopathy

Oral anticoagulation in patients with cerebral amyloid angiopathy is a therapeutic challenge. The association of cerebral amyloid angiopathy with intracerebral hemorrhage, a high mortality of intracerebral hemorrhage especially under oral anticoagulation and the high risk of recurrent bleeding require a multidisciplinary approach and a thorough risk-benefit analysis. Vitamin $\mathrm{K}$ antagonists increase the risk of intracerebral bleeding and the accompanying mortality by $60 \%$ and should be avoided if possible or reserved for special clinical situations (e.g. mechanical aortic valve replacement). Treatment with novel oral anticoagulants and antiplatelet drugs also increases the risk of cerebral bleeding and therefore needs a thorough risk-benefit evaluation. An interventional left atrial appendage closure is a promising therapeutic option especially in patients with an absolute arrythmia with atrial fibrillation.

Furthermore, other clinical implications in patients with cerebral amyloid angiopathy are the subject of this review of the literature, such as special characteristics after acute ischemic stroke and the necessary secondary prophylaxis, with previous intracerebral hemorrhage and in patients with cognitive deficits.

\section{Keywords}

Cerebral amyloid angiopathy · Intracerebral hemorrhage · Platelet inhibition · Oral anticoagulation . Interventional left atrial appendage closure

Open Access. Dieser Artikel wird unter der Creative Commons Namensnennung 4.0 International Lizenz veröffentlicht, welche die Nutzung, Vervielfältigung, Bearbeitung, Verbreitung und Wiedergabe in jeglichem Medium und Format erlaubt, sofern Sie den/die ursprünglichen Autor(en) und die Quelle ordnungsgemäß nennen, einen Link zur Creative Commons Lizenz beifügen und angeben, ob Änderungen vorgenommen wurden.

Die in diesem Artikel enthaltenen Bilder und sonstiges Drittmaterial unterliegen ebenfalls der genannten Creative Commons Lizenz, sofern sich aus der Abbildungslegende nichts anderes ergibt. Sofern das betreffende Material nicht unter der genannten Creative Commons Lizenz steht und die betreffende Handlung nicht nach gesetzlichen Vorschriften erlaubt ist, ist für die oben aufgeführten Weiterverwendungen des Materials die Einwilligung des jeweiligen Rechteinhabers einzuholen.

Weitere Details zur Lizenz entnehmen Sie bitte der Lizenzinformation auf http://creativecommons.org/ licenses/by/4.0/deed.de.

\section{Literatur}

1. Rother J (2020) Stroke and intracerebral hemorrhage under anticoagulation or platelet inhibitionwhen should treatment be restarted and how? Internist (Berl) 61(4):424-430

2. Greenberg SM (2021) Cerebral amyloid angiopathy

3. Pisters R et al (2010) A novel user-friendly score (HAS-BLED) to assess 1-year risk of major bleeding in patients with atrial fibrillation: the Euro heart survey. Chest 138(5):1093-1100

4. Cannistraro RJ, Meschia JF (2018) The clinical dilemma of anticoagulation use in patients with cerebral amyloid angiopathy and atrial fibrillation. Curr Cardiol Rep20(11):106
5. January CT et al (2014) 2014 AHA/ACC/HRS guideline for the management of patients with atrial fibrillation: a report of the American college of cardiology/American heart association task force on practice guidelines and the heart rhythm society. J Am Coll Cardiol 64(21):e1-e76

6. DeSimone CV et al (2017) Cerebral amyloid angiopathy: diagnosis, clinical implications, and management strategies in atrial fibrillation. J Am Coll Cardiol 70(9):1173-1182

7. Kase CS et al (1982) Lobar intracerebral hematomas: clinical and CT analysis of 22 cases. Neurology 32(10):1146-1150

8. Massaro AR et al (1991) Clinical discriminators of lobar and deep hemorrhages: the stroke data bank. Neurology 41(12):1881-1885

9. Grysiewicz R, Gorelick PB (2014) Incidence, mortality, and risk factors for oral anticoagulantassociated intracranial hemorrhage in patients with atrial fibrillation. J Stroke Cerebrovasc Dis 23(10):2479-2488

10. O'Donnell HC et al (2000) Apolipoprotein E genotype and the risk of recurrent lobar intracerebral hemorrhage. N Engl J Med 342(4):240-245

11. Charidimou A et al (2019) Cortical superficial siderosis and recurrent intracerebral hemorrhage risk in cerebral amyloid angiopathy: large prospective cohort and preliminary meta-analysis. Int J Stroke 14(7):723-733

12. Biffi $A$ et al (2015) Association between blood pressure control and risk of recurrent Intracerebral hemorrhage. JAMA 314(9):904-912

13. Biffi A et al (2010) Aspirin and recurrent intracerebral hemorrhage in cerebral amyloid angiopathy. Neurology 75(8):693-698

14. Gurol ME et al (2012) Predicting sites of new hemorrhage with amyloid imaging in cerebral amyloid angiopathy. Neurology 79(4):320-326

15. Charidimou A et al (2019) Cortical superficial siderosis and bleeding risk in cerebral amyloid angiopathy: a meta-analysis. Neurology 93(24):e2192-e2202

16. Vernooij MW et al (2009) Use of antithrombotic drugs and the presence of cerebral micro- 
bleeds: the Rotterdam scan study. Arch Neurol 66(6):714-720

17. Al-ShahiSalman Retal (2019) Effects of antiplatelet therapy on stroke risk by brain imaging features of intracerebral haemorrhage and cerebral small vessel diseases: subgroup analyses of the RESTART randomised, open-label trial. Lancet Neurol 18(7):643-652

18. Shoamanesh A (2020) NOACs for stroke prevention in patients with atrial fibrillation and previous $\mathrm{ICH}$ (NASPAF-ICH)

19. Steiner T, Unterberg A et al (2021) S2kLeitlinie: Behandlung von spontanen intrazerebralen Blutungen. Deutsche Gesellschaft für Neurologie,

20. Ponamgi SP et al (2020) High mortality rates among patients with non-traumatic Intracerebral hemorrhage and atrial fibrillation on antithrombotic therapy are independent of the presence of cerebral Amyloid Angiopathy: insights from a population-based study. J Am Heart Assoc 9(15):e16893

21. Lovelock CE et al (2010) Antithrombotic drug use, cerebral microbleeds, and intracerebral hemorrhage: a systematic review of published and unpublished studies. Stroke 41(6):1222-1228

22. Rosand J et al (2000) Warfarin-associated hemorrhage and cerebral amyloid angiopathy: a genetic and pathologic study. Neurology 55(7):947-951

23. Hart RG, Boop BS, Anderson DC (1995) Oral anticoagulants and intracranial hemorrhage. Facts and hypotheses. Stroke 26(8):1471-1477

24. Ward R et al (2020) Utility of HAS-BLED and CHA2DS2-VASc scores among patients with atrial fibrillation and imaging evidence of cerebral amyloid angiopathy. Mayo Clin Proc 95(10):2090-2098

25. Wilson D et al (2018) Cerebral microbleeds and intracranial haemorrhage risk in patients anticoagulated for atrial fibrillation after acute ischaemic stroke or transient ischaemic attack (CROMIS-2): a multicentre observational cohort study. Lancet Neurol 17(6):539-547

26. Schrag M et al (2021) Left atrial appendage closure for patients with cerebral amyloid angiopathy and atrial fibrillation: the LAA-CAA cohort. TransI Stroke Res 12(2):259-265

27. Sahay S et al (2017) Efficacy and safety of left atrial appendage closure versus medical treatment in atrial fibrillation: a network meta-analysis from randomised trials. Heart 103(2):139-147

28. Reddy VY et al (2017) 5-year outcomes after left atrial appendage closure: from the PREVAIL and PROTECT AF trials. J Am Coll Cardiol 70(24):2964-2975

29. Kuramatsu JB et al (2015) Anticoagulant reversal, blood pressure levels, and anticoagulant resumption in patients with anticoagulation-related intracerebral hemorrhage. JAMA 313(8):824-836

30. Biffi A et al (2017) Oral anticoagulation and functional outcome after Intracerebral hemorrhage. Ann Neurol 82(5):755-765

31. Rosenqvist MN (2020) Prevention of stroke by left atrial appendage closure in atrial fibirillation patients after Intracerebral hemorrhage (STROKECLOSE). http://clinicaltrials.gov/ ct2/show/NCT03186729.Zugegriffen:08Sep 2021

32. Banerjee $G$ et al (2018) Cognitive impairment before intracerebral hemorrhage is associated with cerebral amyloid angiopathy. Stroke 49(1):40-45

33. Nakata-Kudo $Y$ et al (2006) Microbleeds in Alzheimer disease are more related to cerebral amyloid angiopathy than cerebrovascular disease. Dement Geriatr Cogn Disord 22(1):8-14
34. Block F, Dafotakis M (2017) Cerebral amyloid angiopathy in stroke medicine. Dtsch Arztebl Int 114(3):37-42

35. Tsivgoulis $G$ et al (2016) Risk of symptomatic intracerebral hemorrhage after intravenous thrombolysis in patients with acute ischemic stroke and high cerebral mircobleed burden: a meta-analysis. JAMA Neurol 73:675-683

36. Schlemm L et al (2020) Benefit of intravenous thrombolysis in acute Ischemic stroke patients with high cerebral microbleed burden. Stroke 51(1):232-239

37. Braemswig TB et al (2019) Predictors of new remote cerebral microbleeds after IV thrombolysis for ischemic stroke. Neurology 92(7):e630-e638

38. Arima $\mathrm{H}$ et al (2010) Effects of perindopril-based lowering of blood pressure on intracerebral hemorrhage related to amyloid angiopathy: the PROGRESS trial. Stroke 41(2):394-396

39. Amarenco $P$ et al (2006) High-dose atorvastatin after stroke or transient ischemic attack. $N$ Engl J Med 355(6):549-559

40. Westover MB et al (2011) Statin use following intracerebral hemorrhage: a decision analysis. Arch Neurol 68(5):573-579

41. Teoh RJJ et al (2019) Does statin increase the risk of intracerebral hemorrhage in stroke survivors? A meta-analysis and trial sequential analysis. Ther Adv Neurol Disord 12:1756286419864830

42. Sprugel Ml et al (2021) Impact of statins on hematoma, edema, seizures, vascular events, and functional recovery after intracerebral hemorrhage. Stroke 52(3):975-984
Hier steht eine Anzeige. Springer 